\title{
A Numerical Approach to the Solution of Schrodinger Equation for Hydrogen-Like Atoms
}

\author{
Maysoon A. Mahmood
}

\begin{abstract}
A Numerical approach is used to find the energy levels and electron distribution for each subshell in Hydrogen like atoms. This approach used the built in ability of MATLAB to find Eigen values and Eigen ectors. The space around the nucleus is divided in three dimensions into a large number of elements. The wave function in each element, for a specific subshell,was foundto be the Eigen vector of the Hamiltonian operator matrix constructed for the discrete spatial elements. The square of the magnitude of the wave function is plotted in three dimensions to show the distribution of electron around the nucleus. The electron energy for that subshell was found from the corresponding Eigen value.

The results shows very goodfit with analytical methods used to solve Hydrogen-like atoms. This fit includes both energy levels and electron distribution around nucleus.
\end{abstract}

\section{Introduction}

Schrodinger equation for an electron in Hydrogen atom is ${ }^{[1]}$;

$$
\left(-\frac{\hbar^{2}}{2 m} \nabla^{2}+V\right) \psi=E \psi
$$

Where;

$\boldsymbol{V}:$ is the potential energy of the electron

$\boldsymbol{E}:$ is the total energy of the electron $\psi:$ is the wave function of the electron

And is $\quad-\frac{\hbar^{2}}{2 m} \nabla^{2}$ the kinetic energy operator
The $\quad$ above equation can be written as:

$\breve{\mathrm{H}} \Psi=\mathrm{E} \Psi \ldots \ldots(1)$

$$
-\frac{\hbar^{2}}{2 m} \nabla^{2}+V
$$

Where; $\mathrm{H}=$ is the Hamiltonian operator.

Analytical solutions are available in literature ${ }^{[1]}$, which are perfect for Hydrogen-like atoms. But analytical solutions can hardly be developed to solve more complicated atoms. Some numerical solutions are also available ${ }^{[2],[3]}$, but they suffered from huge number of calculations, so they either work in two dimensions only to reduce the number of calculations, losing generality, or reduce the number of spatial elements, losing accuracy. In this paper, we reduced the problem into an Eigen value problem and used the built in ability of MATLAB to find Eigen values and Eigen vectors. This enable ustokeep accuracy by taking large number of spatial elements and to keep generality by working in three dimensions.

Theory:

Using finite difference method, if the functions are taken at discrete points, Ȟwill be a square matrix and $\Psi$ will be a column vector. Then equation (1) is an Eigen value problem, where Eis an Eigen value of the matrix Ȟand $\Psi$ is its associated Eigen vector.

For discrete functions, the one dimensional Laplacian operator can be calculated as follows ${ }^{[3]}$;

$$
\left[\frac{d^{2} \Psi}{d x^{2}}\right]_{n}=\frac{\Psi_{n+1}-2 \Psi_{n}+\Psi_{n-1}}{a^{2}}
$$


Where $a$ is the element size.

Then the Laplacian operator on $\Psi$ (with respect to $\mathrm{x}$ ) will be $\mathrm{be}^{[4]}$;

$\nabla^{2} \Psi_{=-\ldots .(4)}^{1} \quad\left[\begin{array}{cccccc}2 & -1 & 0 & 0 & 0 & 0 \\ -1 & 2 & -1 & 0 & 0 & 0 \\ 0 & -1 & \ddots & \ddots & 0 & 0 \\ 0 & 0 & \ddots & \ddots & \ddots & 0 \\ 0 & 0 & 0 & \ddots & \ddots & -1 \\ 0 & 0 & 0 & 0 & -1 & 2\end{array}\right]\left[\begin{array}{c}\Psi_{1} \\ \Psi_{2} \\ \vdots \\ \Psi_{n} \\ \vdots \\ \Psi_{N}\end{array}\right]$

\section{Tri-diagonal Matrix}

The three dimensional discrete Laplacian operator with respect to $\mathrm{x}, \mathrm{y}$ and $\mathrm{z}$ can be constructed using the Kronecker tensor product of each of the three operator's matrices for each dimension ( $\mathrm{x}, \mathrm{y}$ and $\mathrm{z}$ ) with similar size identity matrices as follows ${ }^{[5],[6]}$;

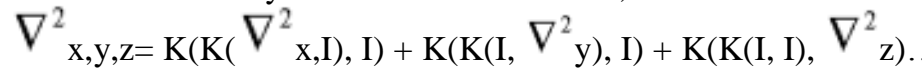

Where $\mathrm{K}(\mathrm{A}, \mathrm{B})$ is the Kronecker tensor product of $\mathrm{A}$ and $\mathrm{B}$.

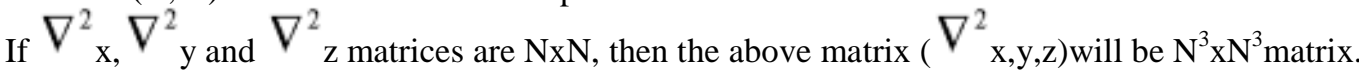

The potential energy varies as $-1 / R$, where $R=\left(x^{2}+y^{2}+z^{2}\right)^{0.5}$, or;

$\mathrm{V}(\mathrm{x}, \mathrm{y}, \mathrm{z})=-1 / \mathrm{R}$

These values should be distributed on the diagonal of an $\mathrm{N}^{3} \mathrm{xN}^{3}$ matrix and then added to the three dimensional discrete Laplacian given by equation (2) to get the Hamiltonian operator $\mathrm{H}$.

So, the Ȟ operator matrix will be;

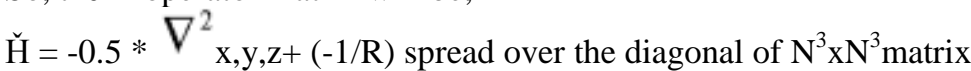

Then the minimum Eigen value of the matrix $\check{\mathrm{H}}$ is the ground level energy of Hydrogen atom (energy of $1 \mathrm{~s}$ orbital) in atomic units. The associated Eigen vector is the wave function $\Psi$ of the $1 \mathrm{~s}$ orbital at each point in space $(\mathrm{x}, \mathrm{y}, \mathrm{z})$. The number of points is $\mathrm{N}^{3}$ and the probability of finding the electron at any point in space is $|\Psi|^{2}$.

The next four minimum Eigen values of the matrix $\mathrm{H}$ are the values of energies of $2 \mathrm{P}_{\mathrm{x}}, 2 \mathrm{P}_{\mathrm{y}}, 2 \mathrm{P}_{\mathrm{z}}$ and $2 \mathrm{~S}$ subshells of the Hydrogen atom. The associated Eigen vectorsare the wave functionsof those four subshells. The next nine minimum Eigen values of the matrix $\breve{H}$ are the values of energies of $3 \mathrm{P}_{\mathrm{x}}, 3 \mathrm{P}_{\mathrm{y}}, 3 \mathrm{P}_{\mathrm{z}}, 3 \mathrm{~S}$, and the five 3 dsubshells of the Hydrogen atom. The associated Eigen vectors are the wave functions of those four subshells.

\section{Results}

The minimum Eigen value of the matrix $\breve{H}$, which is the ground level energy of Hydrogen atom (energy of $1 \mathrm{~s}$ orbital) in atomic units was found. The number of elements was changed to see the effect of that on accuracy, the results are shownin the following table:

\begin{tabular}{|c|c|c|}
\hline $\begin{array}{c}\text { Number of elements "N" in } \\
\text { one dimension }\end{array}$ & $\begin{array}{c}\text { Calculated } \\
\text { Energy }\end{array}$ & error \\
\hline 40 & -0.49134 & $1.7 \%$ \\
\hline 50 & -0.49402 & $1.2 \%$ \\
\hline & & \\
\hline 60 & -0.49549 & $0.9 \%$ \\
\hline 70 & -0.49638 & $0.72 \%$ \\
\hline 80 & -0.49695 & $0.61 \%$ \\
\hline 100 & -0.49763 & $0.47 \%$ \\
\hline 120 & -0.49799 & $0.40 \%$ \\
\hline
\end{tabular}



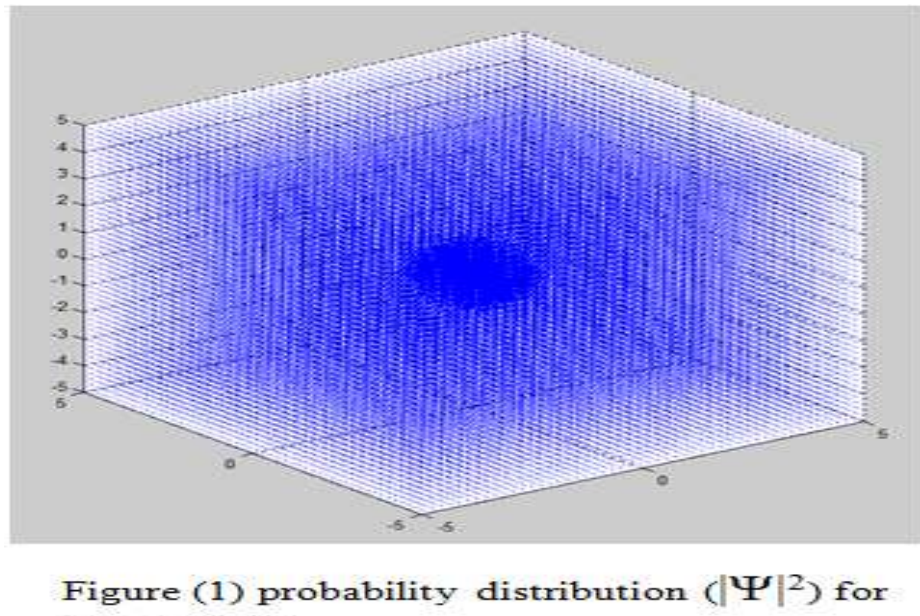

the $1 \mathrm{~S}$ orbital

The results are in good coincidence with the theoretical valueof $(-0.5000)$ and the error is decreasing with increasing number of elements. The space of calculation is 5 units from the nucleus in all directions. The associated Eigen vector, which is the wave function $\Psi$ of the 1 s orbital at each point in space (x, y, z), was also found and the probability of finding the electron at any point in space $\left(|\Psi|^{2}\right)$ is shown in figure (1). This figure shows good coincidence with the figures found in literature ${ }^{[7]}$.

The next four minimum Eigen values of the matrix $\breve{H}$ are found to be equal, they are the energies of the $2 \mathrm{Px}, 2 \mathrm{Py}, 2 \mathrm{Pz}$ and $2 \mathrm{~S}$ respectively. Their calculated values, with $\mathrm{N}=100$, are shown in the following table with theoretical true values for comparison:

\begin{tabular}{|c|c|c|c|}
\hline Subshell & Calculated Value & $\begin{array}{c}\text { Theoretical } \\
\text { Value }\end{array}$ & \% Error \\
\hline $2 \mathrm{Px}$ & -0.1253 & -0.125 & $0.24 \%$ \\
\hline $2 \mathrm{Py}$ & -0.1253 & -0.125 & $0.24 \%$ \\
\hline $2 \mathrm{Pz}$ & -0.1253 & -0.125 & $0.24 \%$ \\
\hline $2 \mathrm{~S}$ & -0.124 & -0.125 & $0.8 \%$ \\
\hline
\end{tabular}

The space of calculation is 15 unitsfrom the nucleus in all directions divided into $10^{6}$ elements $\left(\mathrm{N}^{3}\right)$. The associated Eigen vectors, which are the wave functions of those four subshells, are also found and the figures of the probability of finding the electron at any point in space $\left(|\Psi|^{2}\right)$ are shown in figure $(2 \mathrm{a}-\mathrm{d})$ for $2 \mathrm{P}_{\mathrm{x}}$, $2 \mathrm{P}_{\mathrm{y}}, 2 \mathrm{P}_{\mathrm{z}}$ and $2 \mathrm{~S}$ subshells respectively. These figures show good coincidence with the figures found in literature $^{[7]}$. The difference is that these figures are not aligned with the coordinate axes $\mathrm{x}, \mathrm{y}$ and $\mathrm{z}$. This is normal because the electron distributions in $\mathrm{Px}, \mathrm{Py}$ and $\mathrm{Pz}$ subshells are nominally considered to be aligned with principal axes, butreally, theyare aligned with any three orthogonal axes, which is what these figures show.

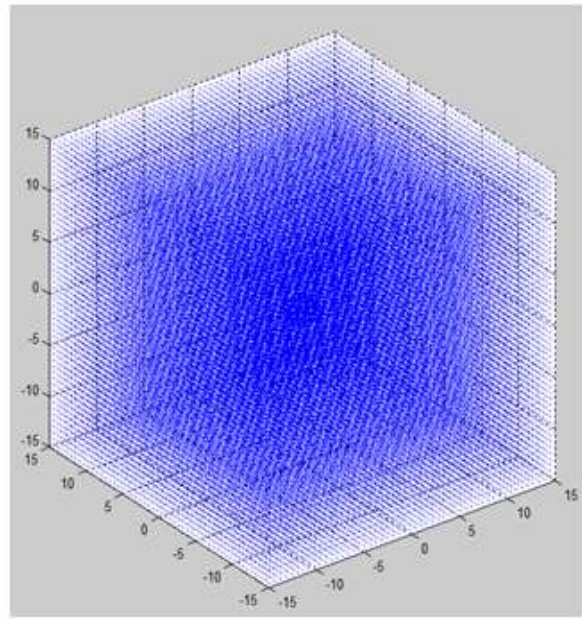

Figure (2-a) probability distribution $\left(|\Psi|^{2}\right)$ for the $2 \mathrm{~S}$ orbital

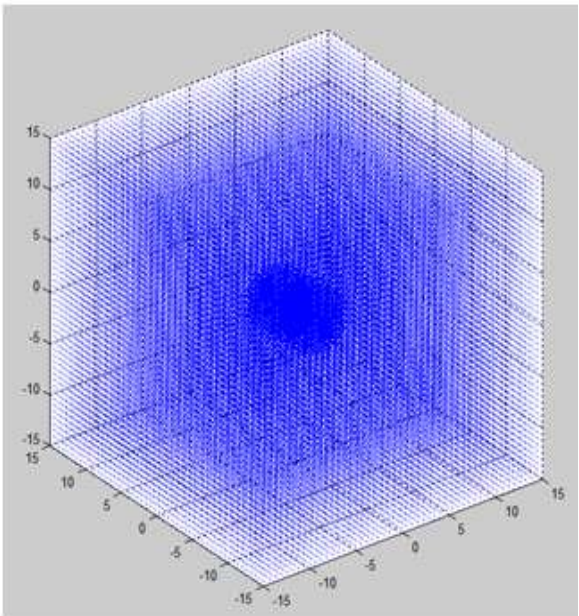

Figure (2-b) probability distribution $\left(|\Psi|^{2}\right)$ for the $2 \mathrm{Px}$ orbital 


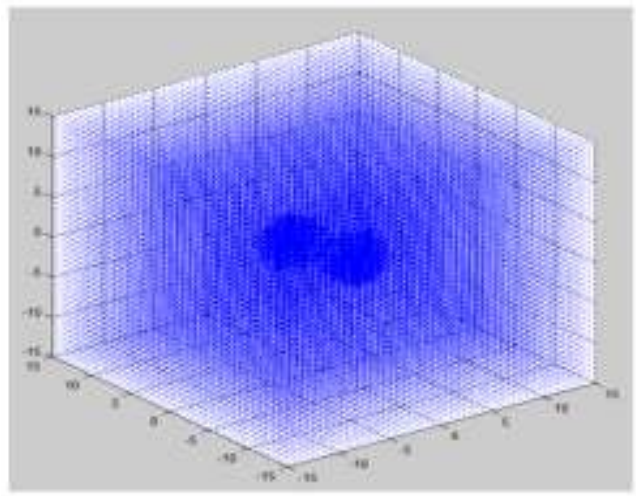

Figure (2-c) probability distribution $\left(|\Psi|^{2}\right)$ for the 2Py orbital

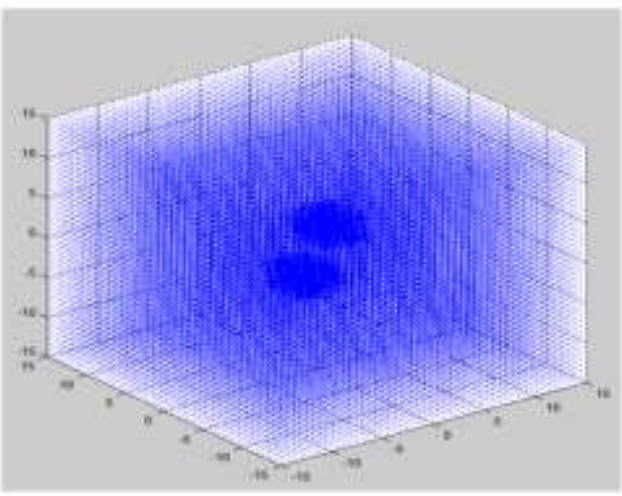

Figure (2-d) probability distribution $\left(|\Psi|^{2}\right)$ for the $2 \mathrm{P}_{2}$ orbital

The next nine minimum Eigen values of the matrix $\mathrm{H}$ are also found to be nearly equal.Their values range from -0.0553 to -0.0532 which are in good coincidence with the theoretical value of $(-0.0556)$. They are the values of energies of $3 \mathrm{P}_{\mathrm{x}}, 3 \mathrm{P}_{\mathrm{y}}, 3 \mathrm{P}_{\mathrm{z}}, 3 \mathrm{~S}$, and five for the $3 \mathrm{~d}$ subshells of the Hydrogen atom.

\section{Conclusion}

The coincidence of energy of electron with theoretical values is great. The error is less than $1 \%$, which is excellent for a numerical solution. The error can also be reduced if the number of elements is increased.

The figures of distribution of electron around the nucleus for all subshells are similar to analytically found distribution figures. They are really better than those figures because they show the distribution in a better more realistic manner.

The great advantage of this method is that it assist the available methods of solutions with good coincidence. It is also possible toevolve this method to find the solutions formore complicated atoms by modifying the potential energy term. Another termshould be added to the potential energy termto take into account the repulsion between electrons inthese atoms. This really need some effort to construct a matrix for this purpose, then the solution will be straightforward.

\section{References}

[1]. Hans A Bethe and Edwin E Salpeter, "Quantum mechanics of one and two-electron atoms" Dover Pub. INC, NY 2008.

[2]. Anders W. Sandvik, "Numerical Solutions of the Schr" odinger Equation", PY 502, Computational Physics, Fall 2015

[3]. SimenKvaal, " A Critical Study of the Finite Differenceand Finite Element Methods for theTime Dependent Schrödinger Equation", PHD thesis, University of Oslo, 2004.

[4]. SupriyoDatta, "Fundamentals of Nano-electronics", Sept 11, 2009, Chapter 2.2

[5]. Wolfgang Hackbusch and Boris N. Khoromskij, "Hierarchical Kronecker Tensor-Product Approximations", Max-PlanckInstitutMathematik in den Naturwissenschaften, Inselstr. 22-26, D-04103 Leipzig, Germany

[6]. Lambert M. Surhone, Mariam T. Tennoe and Susan F. Hensonnow "Kronecker sum of Discrete Laplacians", Betascript Publishing 2010, ISBN-13: 978-613-1-32832-9

[7]. Hans Kuhn, Horst-Dieter Forsterling and David H. Waldeck "Principles of physical chemistry", 2 ${ }^{\text {nd }}$ Edition, 2009, John Wiley. 